\title{
AVALIAÇÃO IN VITRO DO POTENCIAL CITOTÓXICO DOS ÓLEOS DE ALPINIA SPECIOSA, AGERATUM CONYZOIDES E DYSPHANIA AMBROSIOIDES EM LINHAGEM CELULAR DE CÂNCER DE PRÓSTATA, LINFOMA HISTIOCÍTICO E LEUCEMIA MIELOIDE CRÔNICA
}

\author{
V.A.S. SILVA $^{1}$, M. A. B. CORREIA ${ }^{1}$, M. J. B. M. RÊGO ${ }^{1}$, M. C. PEREIRA ${ }^{1}$, R. D. \\ $\mathrm{SA}^{2}$, R. F. SANTOS ${ }^{2}$, T. G. J. SILVA ${ }^{2}$, K. P. RANDAU ${ }^{2}$, I. R. PITTA ${ }^{1}$, M. G. R. \\ PITTA $^{1}$
}

\begin{abstract}
${ }^{1}$ Universidade Federal de Pernambuco, Centro de Biociências, Laboratório de Inovação Terapêutica (LINAT), Núcleo de Pesquisa em Inovação Terapêutica (NUPIT) ${ }^{2}$ Universidade Federal de Pernambuco, Centro de Ciências da Saúde, Departamento de Ciências Farmacêuticas, Laboratório de Farmacognosia,
\end{abstract} E-mail para contato: vitinhoalfredo85@hotmail.com

\begin{abstract}
RESUMO - O câncer é um problema de saúde pública que tem afetado milhões de pessoas e causado altas taxas de óbito em todo mundo. Diante disso, o objetivo do trabalho foi avaliar o efeito citotóxico do óleo das folhas de A. speciosa, A. conyzoides e D. ambrosioides em linhagem celular de Próstata, Linfoma histiocítica e Leucemia mieloide crônica. Para isso, realizamos o ensaio de citotoxicidade pelo método do MTT em linhagem de $D U-145$, U-937 e K-562. Os resultados indicam que na linhagem que K-562, os óleos da folha de A. speciosa, A. conyzoides e D. ambrosioides apresentaram citotoxicidade a partir das doses, $2,5 \times 10^{-3} \%\left(I_{50} 1,7 \times 10^{-3} \%\right)$, $8 \times 10^{-3} \% \quad\left(I C_{50} \quad 5 \times 10^{-3} \%\right)$ e $5 \times 10^{-3} \% \quad\left(I C_{50} 3 \times 10^{-2} \%\right)$, respectivamente. Enquanto, na linhagem U-937, apenas o óleo de D. ambrosioides foi

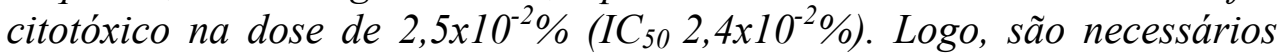
mais estudos para avaliar os mecanismos de ação em linhagem de leucemia mieloide crônica e da espécie D. ambrosioides em linhagem de linfoma histiocítica.
\end{abstract}

Palavras chaves: Citotoxidade, plantas medicinais, antitumoral

ABSTRACT - Cancer is a public health problem that has affected millions of people and caused high death rates worldwide. Therefore, the objective of this work was to evaluate the cytotoxic effect of A. speciosa, A. conyzoides and D. ambrosioides leaves on the Prostatic cell line, histiocytic lymphoma and chronic myeloid leukemia. For this, we performed the cytotoxicity assay by the MTT method in line of DU-145, U-937 and K-562. The results indicated that in the lineage K-562, leaf oils of A. speciosa, A. conyzoides and D. ambrosioides showed cytotoxicity from the doses, $2.5 \times 10^{-3} \%\left(I C_{50}\right.$ $\left.1.7 \times 10^{-3} \%\right), \quad 8 \times 10^{-3} \% \quad\left(I C_{50} \quad 5 \times 10^{-3} \%\right)$ and $5 \times 10^{-3} \% \quad\left(I C_{50} \quad 3 \times 10^{-2} \%\right)$, 
respectively. While in the U-937 strain, only D. ambrosioides oil was cytotoxic in the dose of $2.5 \times 10^{-2} \%\left(I C_{50} 2.4 \times 10^{-2} \%\right)$. Therefore, further studies are needed to evaluate the mechanisms in chronic myeloid leukemia and D. ambrosioides lineage in histiocytic lymphoma.

KeyWords: Cytotoxicity, medicinal plants, antitumor.

\section{INTRODUÇÃO}

O câncer é um problema de saúde pública que tem afetado milhões de pessoas em todo mundo. Entre os diversos tipos de câncer, o de próstata figura como o segundo tipo mais comum entre os homens e representa $6,6 \%$ do total de óbitos masculinos. A Leucemia está entre o sexto e sétimo cancer mais frequente na Região Norte, Sul e Sudeste do Brasil entre homens e mulheres. Só no ano de 2012 foi estimado 265 mil óbitos no mundo. Linfoma histiocítico é um tipo de linfoma não Hodgkin (LNH), raro e muito agressivo. No ano de 2012, LNH foi responsável por 200 mil óbitos (INCA, 2016).

O Brasil possui grande potencial natural e tem investido em políticas públicas para o uso de plantas medicinais dentro do sistema de saúde. Em virtude disso, pesquisas com substratos naturais provenientes de plantas têm despertado grande interesse na busca por tratamento para diversos tipos de doenças, incluindo o câncer (GREENWELL; RAHMAN, 2015). Alpinia speciosa (Zingiberaceae) difundida na região do Nordeste do Brasil, apresenta propriedades anticâncer em linhagens de câncer de pulmão (TAIRA; NGUYEN; TAWATA, 2017). Dysphania ambrosioides (Amaranthaceae) com ampla distribuição no mundo e nativa da América Central e do Sul e possui um amplo espectro de atividade biológica e antitumoral (OLIVEIRA et al., 2014; SÁ et al., 2015). Ageratum conyzoides (Asteraceae) bastante difundida na região tropical e subtropical do mundo, possui ação anti-inflamatória, fungicida e inseticida, e atualmente sua ação antitumoral vem sendo relatada na literatura (MOMESSO et al., 2009). Diante do exposto, o objetivo desse estudo foi avaliar o efeito citotóxico dos óleos da folha de Alpinia speciosa, Ageratum conyzoides e Dysphania ambrosioides em linhagens tumorais de próstata (DU145), linfoma histiocítico (U-937) e leucemia mieloide crônica (K562).

\section{MATERIAIS E MÉTODOS}

2.1. Cultivo de células tumorais: As linhagens de câncer de próstata (DU-145), linfoma histiocítica (U-937) e leucemia mieloide crônica (K-562), foram obtidas do Banco de Células do Rio de Janeiro (BCRJ) e mantidas à $37^{\circ} \mathrm{C}$ com $5 \%$ de $\mathrm{CO}_{2}$ em meio RPMI1640 (U-937 e K-562) e DMEM (DU-145) com 3,7g/L de bicarbonato de sódio, 1\% penicilina e estreptomicina, suplementado com $10 \%$ de soro fetal bovino.

2.2. Ensaio de viabilidade celular por MTT: As células de linhagem tumoral foram plaqueadas na concentração de $10^{4}$ (DU-145), $5 \times 10^{3}$ (K-562) e $5 \times 10^{5}$ (U-937) em placas de 96 poços. Os óleos brutos das folhas das plantas (A. speciosa, A. conyzoides e D. ambrosioides) nas porcentagens de $10^{-3} \%, 2,5 \times 10^{-3} \%, 5 \times 10^{-3} \%, 8 \times 10^{-3} \%, 1 \times 10^{-2} \%$, $2,5 \times 10^{-2} \%$ e $5 \times 10^{-2} \%$ foram utilizados para o tratamento das células no período de 72 horas. As placas foram mantidas em estufa à $37^{\circ} \mathrm{C}$ e $5 \%$ de $\mathrm{CO}_{2}$. Após este período foi adicionado $20 \mu \mathrm{L}$ de MTT (3-(4,5-dimetiltiazol-2yl)-2,5-difenil brometo de tetrazolina), após 3 horas de incubação $130 \mu \mathrm{L}$ SDS (dodecil sulfato de sódio) e mantidas em 
temperatura ambiente por 24 horas. A leitura foi realizada em leitor de microplacas (Biotek EL808) em 570nm. Os experimentos foram realizados em triplicata.

\section{RESULTADOS E DISCUSSÃO}

Resultados anteriores mostraram que os óleos estudados não apresentaram citotoxicidade em PBMC de voluntários saudáveis, exceto os óleos de $A$. speciosa e $D$. ambrosioides na concentração de $5 \times 10^{-2} \%$. Nas análises do óleo da espécie $A$. speciosa, observa-se citotoxicidade apenas na linhagem de leucemia mieloide crônica na dose $2,5 \times 10^{-3} \%$ (viabilidade celular $16,72 \%$ e $\mathrm{IC}_{50} 1,7 \times 10^{-3} \%$ ). Para o óleo da folha de $A$. conyzoides, observa-se citotoxicidade apenas na linhagem de próstata na dose $5 \times 10^{-2} \%$ (viabilidade celular $23,77 \%$ e $\mathrm{IC}_{50} 3,7 \times 10^{-2} \%$ ) e leucemina mieloide crônica a partir da dose $8 \times 10^{-3} \%$ (viabilidade celular $41,30 \%$ e $\mathrm{IC}_{50} 5 \times 10^{-3} \%$ ). No óleo de $D$. ambrosioides, a citotoxicidade na linhagem de leucemia mieloide crônica foi na concentração de $5 \times 10^{-}$ ${ }^{3} \%$ (viabilidade celular $25,57 \%$ e $\mathrm{IC}_{50} 3 \times 10^{-2} \%$ ) e em linfoma histiocítica na dose $2,5 \times 10^{-}$ ${ }^{2} \%$ (viabilidade celular $45,77 \%$ e $\mathrm{IC}_{50} 2,4 \times 10^{-2} \%$ ) (Figura 1 ).

Figura 1. (A) Efeito citotóxico dos óleos de A. speciosa, A. conyzoides e D. ambrosioides em linhagem tumoral de câncer de próstata (DU-145), (B) linhagem de leucemia mieloide crônica (K-562) e (C) câncer de linfoma histiocítico (U-937)

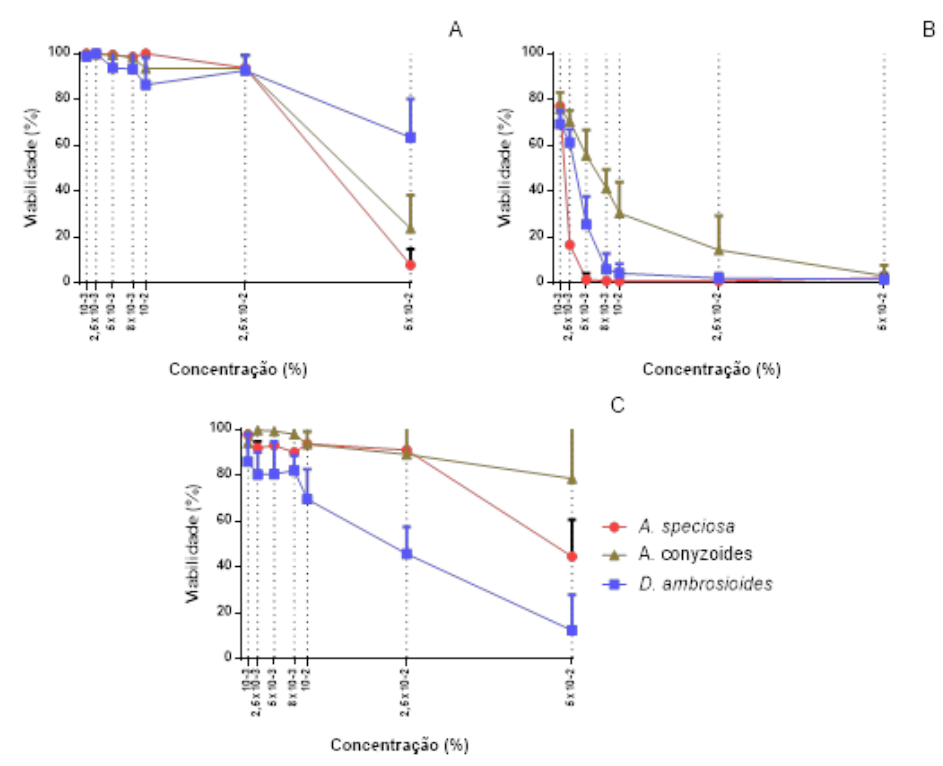

Compostos bioativos de $A$. speciosa têm sido referido por apresentarem bloqueadores de PAK-1, uma proteína chave associada a vários tipos de doenças incluindo o câncer. E por isso, atualmente tem sido extensivamente pesquisada como estratégia para a terapia do câncer (TAIRA; NGUYEN; TAWATA, 2017; NGUYEN at al., 2016). Estudos utilizando o extrato de $A$. conyzoides têm sido referido por apresentar efeito antitumoral em linhagens cancerígenas de carcinoma de pulmão humano (A-549), leucemia em rato (P-388) e humano (Jurkat) (MOMESSO et al., 2009; ACHEAMPONG et al., 2015). Segundo a literatura o óleo essencial de $D$. ambrosioides, apresenta atividade citotóxica em linhagem de câncer de mama (MCF-7) e corroborando com nosso estudo, em leucemia mieloide crônica (K-562) (JIA-LIANG et al., 2013; DEGENHARDT et al., 2016). 


\section{CONCLUSÃO}

INOVAÇÃO TERAPÊUTICA

Os óleos de $A$. speciosa, A. conyzoides e D. ambrosioides, mostraram boa atividade citotóxica, em baixas doses, na linhagem de leucemia mieloide crônica (K562). Além disso, o óleo da espécie $D$. ambrosioides também foi citotóxica na linhagem de linfoma histiocítica na concentração de $2,5 \times 10^{-2} \%$. Logo, são necessários mais estudos para avaliar os mecanismos de ação dos óleos das três espécies, em linhagem de leucemia mieloide crônica e da espécie D. ambrosioides em linhagem de linfoma histiocítica.

\section{REFERÊNCIAS}

ACHEAMPONG et al., In vitro Antioxidant and Anticancer Properties of HydroethanolicExtracts and Fractions of Ageratum conyzoides. EJMP. v. 7, p. 205-214, 2010.

GREENWELL; RAHMAN. Medicinal Plants: Their Use in Anticancer Treatment. Int J PharSciRes. v. 6, p. 4103-4112, Novembro, 2015.

MINISTÉRIO DA SAÚDE. Estimativa/2016 - incidência de Câncer no Brasil. Rio de Janeiro: Editora Coordenação de prevenção e vigilância, 2015.

MOMESSO L.S, Atividade antitumoral do Ageratum conyzoides L. (Asteraceae). Revista Brasileira de Farmacognosia. v. 19, p. 660-663, Setembro, 2009.

NGUYEN at al., Artepillin C and Other Herbal PAK1-blockers: Effects on Hair Cell Proliferation and Related PAK1-dependent Biological Function in Cell Culture. Phytother Res. V. 30, p. 120-127 2016.

OLIVEIRA; FERREIRA; BARROSO. Herb Santa Maria (Chenopodium ambrosioides

L.): Clinical applications and toxic forms - A review. v. 7, p. 464-499, 2014.

SÁ t al., Óleo essencial de Chenopodium ambrosioides L.: estado da arte, Journal of

Basic andApplied Pharmaceutical Sciences, v. 36, p.267-276, 2015.

TAIRA; NGUYEN; TAWATA, Hair Growth Promoting and Anticancer Effects of p21activated kinase 1(PAK1) Inhibitors Isolated from Different Parts of Alpinia zerumbet. Molecules. v. 22, 2017.

\section{AGRADECIMENTOS}

FACEPE, CAPES, INCT_if, LINAT e Laboratório de Farmacognosia. 